\title{
ESTIMATION OF ULTRASONIC ATTENUATION AND MEAN BACKSCATTERER SIZZE VIA DIGITAL SIGNAL PROCESSING.
}

\author{
C. R. Meyer ${ }^{1}$, D. S. Herron ${ }^{2}$, P. L. Carson', \\ R. A. Banjavic ${ }^{3}$, G. A. Thieme ${ }^{4}$, F. L. Bookstein ${ }^{1}$, and M. L. Johnson ${ }^{5}$. \\ ${ }^{1}$ Department of Radiology, Box 13, University of Michigan Hospitals, Ann Arbor, MI 48109 \\ ${ }^{2}$ Radiation Oncology Center, Harper-Grace Hospital, Detroit, MI 48201 \\ ${ }^{3}$ Rocky Mountain Medical Physics, 2851 S. Parker Rd., Suite 920, Aurora, Co 80014 \\ ${ }^{4}$ Department of Radiology, Vanderbilt University, Nashville, TN 37203 \\ ${ }^{5}$ Department of Radiology, University of Colorado, Denver, CO 80262
}

The backscattered if signals from the lungs of fetal sheep during their last trimester of development were digitized and processed in an attempt to correlate ultrasonic parameters with measured functional parameters related to lung maturation. The broadband, post-TGC, if signal of a commercial B-mode ultrasonic scanner was digitized at a sampling rate of $25 \mathrm{MHz}$. Sorting excluded data from regions of rib shadowing and other nonlung structures from analysis. The sorted data were used to estimate the slope of the ultrasonic attenuation coefficient with respect to frequency via linear regression on the average difference of the logarithm of power spectra from separated data segments. The power spectra were also corrected for attenuation, averaged and used to compute the power cepstrum of the backscattered signal which can be related to mean backscatterer radius. Results are presented for eight fetal sheep.

Key words: Attenuation coefficient; backscatter; fetal lung maturity; tissue characterization; ultrasound.

\section{INTRODUCTION}

Every year there are approximately 50,000 premature births in the United States, all having elevated risk of neonatal problems. In practice, pregnancies at risk of premature delivery are continued until the risk of neonatal death from immaturity falls below the risk of serious maternal or fetal damage. Of the diseases of prematurity, the most common is Idiopathic Respiratory Distress Syndrome (IRDS), a specific form of functional immaturity of the lungs. Surface-active material in the alveolar cells is required to maintain alveolar opening after the newborn begins to breathe air; its synthesis, storage and release from the alveolar cell is an essential part of normal fetal lung maturation. The recognition of IRDS as the consequence of insufficient surfactant in the alveoli led to the search for a prenatal assay for it.

Since the work of Gluck and Kulovich in the early 1970s [1], the more common tests for lung function have analyzed anmiotic fluid from amniocentesis with various biophysical or electrophoretic techniques. The lecithin/sphingomyelin (L/S) ratio is the current standard technique, and when low, is moderately pathognomonic for IRDS. Quantitation of other phospholipids improves upon the accuracy of this predictor considerably. For instance, the presence or absence of phosphatidylglycerol (PG) is both more sensitive and more specific as an indicator of fetal lung maturity. Likewise, criteria based on the concentrations of disaturated phosphatidylcholine [2] are both more sensitive and more specific than the L/S ratio. Particular criteria are being designed for meconiumcontaminated samples, samples from diabetic mothers, etc. As the two-dimensional gel techniques become more widely available, clinicians are better able to estimate lung maturity in fetuses which would certainly be considered immature on other, nonpulmonary criteria. But there are patent clinical risks involved in amniocentesis, and lower risk tests would be preferred. If ultrasound also can predict when the lung will mature or will be able to respond to stimulants for surfactant production, it would fill a unique role and 
considerably improve the perinatal management of premature deliveries. A noninvasive method to identify structural properties of the fetal lung which predict future functional maturity would be an important advance in prenatal pulmonary diagnosis. Uttrasound cannot measure any chemical parameter of fetal lung maturity, such as the phospholipids, nor can pulse echo ultrasound provide direct histologic or physiologic information about fetal lung maturity in vivo. However, parameters derived from a quantitative analysis of sound scattered by lung parenchyma may express the changing morphologic pattern of the lung during fetal development, and thereby indicate maturity indirectly via structure. For example, should surfactant production significantly alter fluid retention in the lung, that change in lung characteristics at the precise onset of maturity might engender a sudden, easily detected change in ultrasound scattering.

\section{METHODS}

To determine if ultrasound might be developed as a noninvasive test for direct assessment of maturity of the fetal lung, fetal sheep lungs were scanned with a high quality diagnostic ultrasound system under the most ideal acoustic conditions for visual and quantitative evaluation of ultrasound interactions. The fetal lamb was selected for these feasibility studies since it has been used extensively in pulmonary physiology research as the best analog to human lung development [3-5]. The fetal lamb is similar in size to the human fetus and the lung progresses through the same stages of development, though the time frame is scaled down and the relative time varies in different stages. Each maternal ewe and fetus were anesthetized and the fetus was delivered by Cesarean section into a $37^{\circ} \mathrm{C}$ isotonic saline bath. Sonographic examination of the living fetal lamb was performed with the lamb still attached by its long umbilical cord. With the intercostal spaces and the lung always in the focal zone of relatively strongly focused transducers, quantitative changes in ultrasound interactions in the lung and visual sonographic changes of lung with respect to liver were compared to gestational age and to surface tension and pressure volume measurements on the lungs which were excised after the ultrasound data was acquired. These last two physiologic tests provided a direct measure as to whether the lung was functionally mature [5,6]. For simplicity, pulmonary maturity was defined as the ability of the lungs to inflate at less than bursting pressure, $30 \mathrm{~cm}$ of water. Results from two groups of fetal lambs are reported: a term group (142-147 days) of physiologically mature lungs capable of sustaining life; and a middle group (120-130 days) of physiologically immature lungs near the transition region.

Controlled single sweep linear scanning was accomplished by attaching the end of the scanning arm of a modified B-scanner to a ball-bearing slide on a steel scanning rod. This scanning rod was attached to the ultrasound unit's scanning arin support so that the scan plane could be tilted, translated, and rotated. Lung scattering, where clearly visible between the rib shadows, was not affected significantly by the presence of the ribs.

\section{Visual Interpretation}

A thorough review of the qualitative conclusions of this study is published elsewhere and will only be summarized here [7]. Visual evaluation of the images was based upon three criteria used for comparison of scattering from lung and liver

A. Attenuation: semi-quantitative evaluation of attenuation in $\mathrm{dB} / \mathrm{cm}$ was estimated by adjustment of the time gain compensation (TGC) slope dial on the Philips (2722 S. Fairview Street, Santa Ana, CA 92704) B-mode scanner, Rohnar Model 5580, until uniform brightness throughout the depth of tissue was seen. The 10 turn slope dial setting could be related to attenuation since the Philips scanner has a carefully controlled exponential TGC, includes compensation for transducer focusing, and utilizes narrowband fitters in the receiver.

B. Relative Scattering Amplitude: determination of lung echogenicity relative to liver was based upon a visible lung to fiver difference in transmissivity of white on black films.

C. Texture: coarse texture meant the presence of large, strong scatterers in a nonuniform pattern; fine texture meant the presence of low level scatterers in a homogeneous 
pattern. Texture assessments were interpreted cautiously because of their gain and TGC dependence, and there is still some question as to whether the texture differences observed could be measured objectively independent of signal level.

of 19 sheep experiments acceptable data for visual interpretation were obtained from 15 studies. Images were obtained at 3.5 and $5.0 \mathrm{MHz}$.

A preliminary report of the ultrasonic appearance of human fetal lung has stated that the reflectivity of lung is equal to or below the level of liver throughout most of pregnancy and that this ratio may reverse in late gestation [6]. Our fetal lamb data obtained under carefully controlled conditions show a different and quite opposite pattern. To our knowledge, there is no significant species variation between lambs and humans to account for the difference. In summary, we found that lung echogenicity less than or equal to liver, and lung texture equal to liver was indicative of mature lungs. Lung echogenlcity greater than liver (by at least $2 \mathrm{~dB}$ ) and lung texture coarser than liver was an immature sonographic pattern. A third transitional sonographic pattern included those lambs demonstrating intermediate echogenicity and texture patterns.

\section{Quantitative Analysis}

The Philips B-mode scanner was modified by the vendor to allow switch selectable bypass of existing filter traps in the receiver to obtain a broadband, time gain compensated (TGC), radio frequency ( $r$ ) output. The selected ultrasonic disk transducer had a nominal center frequency of $5 \mathrm{MHz}$, a 54 percent fractional bandwidth, a $19 \mathrm{~mm}$ diameter face and was focused at $9 \mathrm{~cm}$. The broadband, post-TGC if output from the Philips scanner was digitized. The digitizer was a microprocessor controlled device capable of remote programming from the host minicomputer via an EIA, RS 232,9600 baud port. The Precision Data Systems (PDS) digitizer was built according to our specifications using an 8 bit TRW flash digitizer chip, Intel 8086 microprocessor with 8 KBytes of memory, and operated at a sampling rate of $25 \mathrm{MHz}$ with full 8 bit significance [8]. The digitizer's acquisition cycle was triggered by the digital signal transition in the scanner which triggered its pulser. After triggering, the digitizer was programmed to delay acquisition for $\mathrm{T} 1 \mu$ s where $\mathrm{T} 1$ was chosen to correspond to the beginning of backscattered data from inside the chest wall of the fetus. The digitizer acquired a data record of T $2 \mu s$ duration where T2 was long enough to include all backscattered lung data of interest (a maximum of $62 \mu \mathrm{s}$ corresponding to $4.8 \mathrm{~cm}$ of tissue). To minimize quantization error the TGC and system gain were adjusted such that the low level backscattering signal filled the 1 volt infut window of the digitizer. The contents of the digitizer's full memory were dumped to the host memory via a parallel transfer, direct memory access interface at $10 \mathrm{C}$ $\mathrm{KBytes/s}$. The parailel lines of $\mathrm{rf}$ data were acquired in a spatially ordered sequence by allowing the digitizer to be triggered only after a sufficient $x$-axis motion increment in the scanner's arm. By computing and sequentially displaying the envelope of the digitized if data on adjacent lines of the minicomputer's digital scan converter, a crude Bmode echogram of the data acquisition region was generated. The computed B-mode display was used to sort the data to exclude large, nonalveolar structures such as the heart, rib shadows, body walls, large arteries, etc. from further computed analysis. Sorting was performed somewhat subjectively by an investigator's marking of the regions of interest via a keyboard cursor displayed on the computed B-mode image (see Fig. 1). These sorted files were used to compute parametric estimates of lung backscattering.

Time gain compensation (TGC) is beneficial in that the quantization errors of the digitizer can be minimized over the whole input record. This is especially important for smaller bit size converters where the dynamic range is already limited, i.e. $48 \mathrm{~dB}$ for a true 8 bit converter. Functionally $T G C$ is the multiplication of an exponential signal with the input rf signal. In the frequency domain the result is the convolution of the Fourier transforms of the rf signal and the exponential TGC signal. Since the Fourier transform of the TGC signal used to compensate for tissue having an attenuation of 0.5 $\mathrm{dB} / \mathrm{cm} / \mathrm{MHz}$ insonified with a $5 \mathrm{MHz}$ transducer has an upper $-3 \mathrm{~dB}$ cutoff frequency of $7 \mathrm{kHz}$, the convolution process will smooth only very fine details of the broadband back scattered rf spectrum and will not adversely affect broadband analytic techniques. in fact, the use of short duration data windows often has a more significant smoothing effect than TGC. 


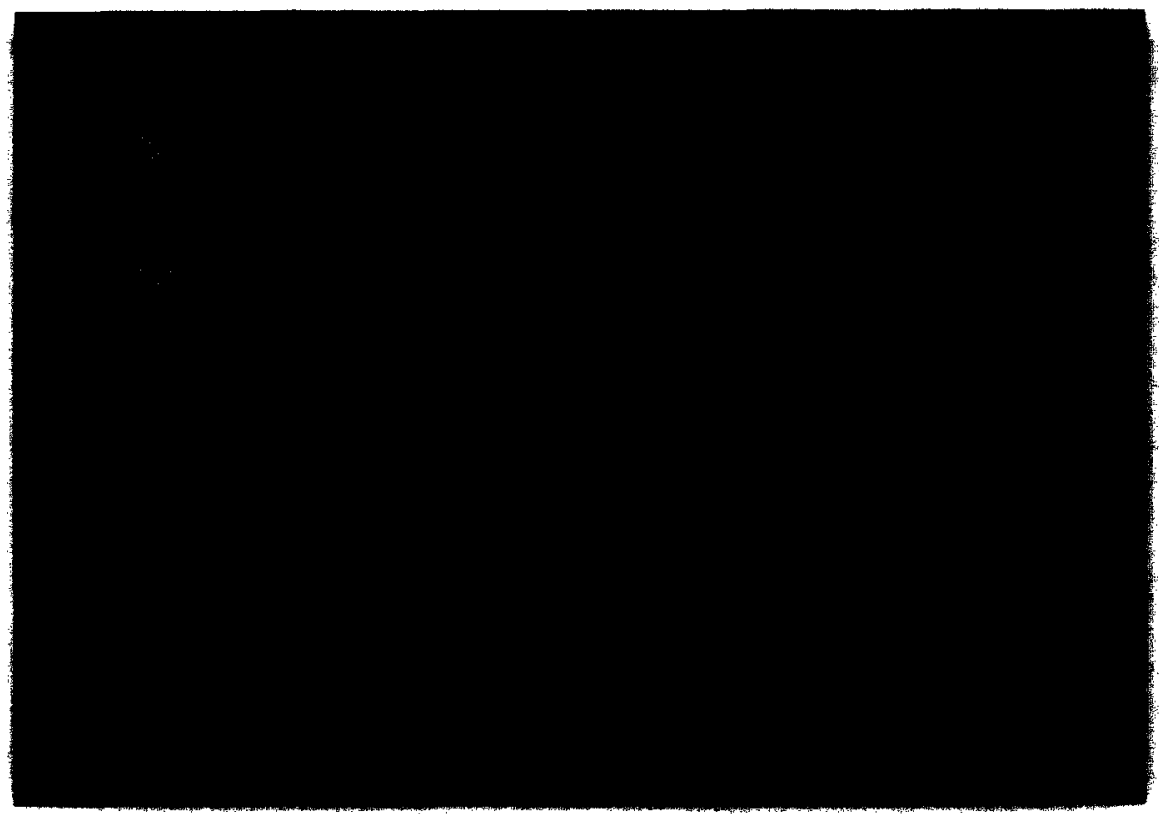

Fig. 1 B-mode echogram computed via envelope detection of digitized rf data. Lines of sorted if data lie between bright pixels.

Two quantitative parameters which can be computed from ultrasonic backscattering which could be correlated with other measures of fetal lung maturity were $\alpha$, the slope of the attenuation coefficient with respect to frequency, and the mode of the backscattered power cepstrum. Both parameters are insensitive to amplitude effects such as system gain and TGC settings since both depend on the shapes of the backscattered spectra rather than on absolute amplitudes. Such would not be the case for amplitude histograms of the envelope detected rf signal. The data interval between the region of interest boundaries along any digitized line of backscatter was divided into overlapping, 128 point segments where the overlap was 64 points (Fig. 2). The modified technique of averaging power spectra from overlapping segments has been shown to reduce the variance of the estimates for a large number of segments to 61 percent of that obtained with non-overlapping segments of the same length $[9,10]$. The assumed frequency domain model of backscattering from each data segment is given by

$$
E\left[P_{n}(f)\right]=g_{n} \quad e^{-4 b f} \quad T^{2}(f) \quad B(f) e^{4 a n \Delta x f}
$$

where $E\left[P_{n}(f)\right]$ is the estimate of the received power spectrum of the $n$th segment for $\mathrm{n}=0,1,2, \ldots, \mathrm{N}, g_{n}$ is the system gain including TGC, $e^{-4} b f$ is the effect of intervening tissue between the transducer and the first segment, $T^{2}(f)$ is the round trip spectral effects of the transducer, $B(f)$ is the backscattered power spectrum from the segment, $\alpha$ is the attenuation coefficient of the lung, and $\Delta x$ is $1.97 \mathrm{~mm}$.

Since the round trip spectral content of the wave changes with range due to diffraction, we identified a region in the focussed beam where the diffraction effects were insignificant. The region was $4.8 \mathrm{~cm}$ long and began just distal to the transducer's focal point. The region was verified in two ways. A stepless gate and analog spectrum analyzer were used to examine the echo from a flat, perpendicular sylastic rubber target in degassed water; no changes in spectral shape were observed as the target was manually moved through the $4.8 \mathrm{~cm}$ region. In addition, the backscatter from a uniform scattering phantom manufactured by Radiation Measurements Inc (RMI, P.O. Box 327 , Middleton, WI 53562) was digitized; computed attenuation coefficients from this data 


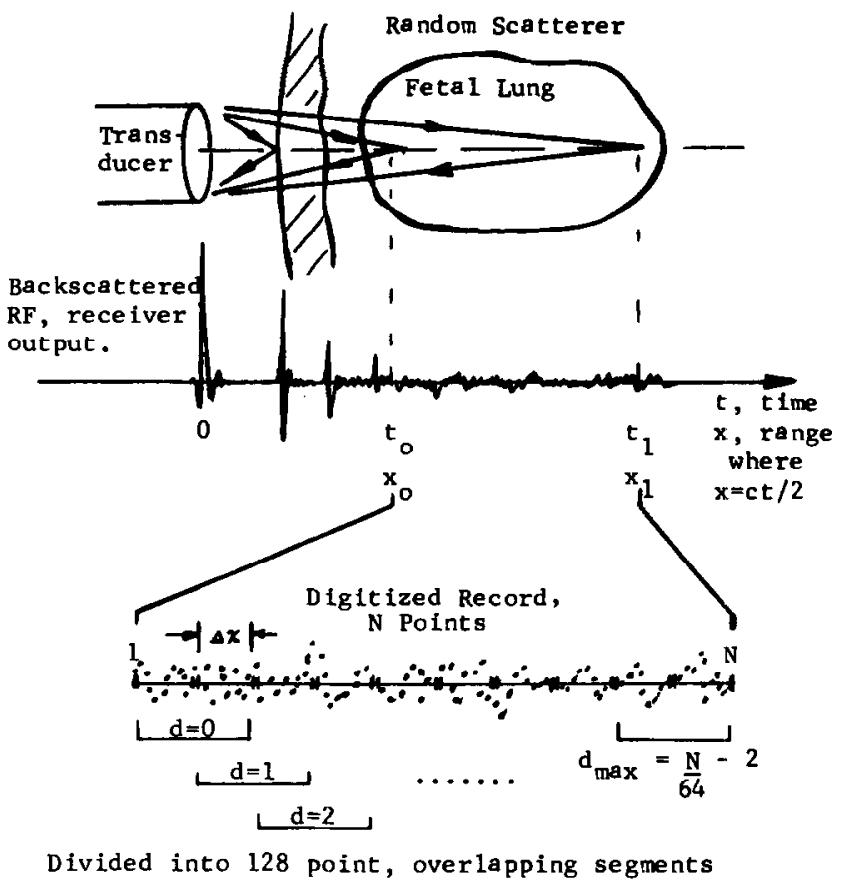

Fig. 2 Relationship of overlapping data segments to physical setting of experiment.

did not vary significantly with range. During scanning and data acquisition, the transducer was positioned such that the focal point was near the chest wall and the region of uniform spectral content was within the fetal lung.

Kuc has thoroughly described the technique of averaging the difference of log spectra from data segments separated by the interval, $d$, to obtain the mimimum variance estimate of the slope of the attenuation coefficient with respect to frequency by choosing $d=2 / 3$ of the total length available [11]. We implemented the fundamentals of Kuc's technique except that no adaptive length data windows were used. Each segment was windowed with a 128 point Hamming window before taking the fast Fourier transform. The global estimate of $\alpha$ for the fetal lung was computed from a weighted sum of the individual estimates of each line. The weight for each line included in the average was inversely proportional to the a priori variance estimate. This technique prevents bad estimates, i.e. ones picked from distributions of large variance, from corrupting good estimates and results in the global least mean square error estimate. According to Kuc's derivation, the a priori variance of the estimate is inversely proportional to the cube of the total length of the data segment. Thus, the global estimate of $\alpha$ is the sum of estimates for each line weighted by a factor proportional to the cube of the line length. The effects of experimental variance may be seen in table 1 which reports the experimental standard error of the mean for each set of line lengths encountered in the sorted data set as well as the global estimate and its standard error. The results of computing $\alpha$ for eight fetal sheep is shown in figure 3 where $\alpha$ is plotted vs. the minimum surface tension measurement made from several postmortem biopsy minces of the fetal lung. The normalized correlation coefficient in figure 3 is 0.83 . Note that fetal sheep $L$, an apparent outlier, was meconium stained and visibly growth retarded at delivery, although it was full term ( 145 days gestation). If $L$ were excluded from the regression analysis, the correlation coefficient would increase to 0.96 , although it is not worth making much of this point for a study size of only eight.

In addition to estimating the attenuation coefficient, we estimated another parametric measure of backscatter, the mode of the backscattered power cepstrum. The rationale for use of the cepstrum is based on modeling backscatter from the fetal 
MEYER ET AL.

TABLE 1: EXPERIMENTAL PERFORMANCE OF ATTENUATION ESTIMATOR

\begin{tabular}{c|c|c|c}
\hline $\begin{array}{c}\text { Number of } \\
\text { Overlapping } \\
\text { Segments }\end{array}$ & $\begin{array}{c}\text { Number } \\
\text { of } \\
\text { Lines }\end{array}$ & $\begin{array}{c}\text { Mean } \\
\text { (dB/cm MHz) }\end{array}$ & $\begin{array}{c}\text { Standard } \\
\text { Error of } \\
\text { Mean }\end{array}$ \\
\hline \hline 3 & 13 & -0.81 & 1.82 \\
5 & 182 & 0.21 & 0.42 \\
7 & 296 & 0.63 & 0.21 \\
9 & 242 & 0.58 & 0.18 \\
11 & 206 & 0.53 & 0.14 \\
13 & 42 & 0.45 & 0.16 \\
19 & 11 & 0.46 & 0.12 \\
\hline \multicolumn{2}{r|}{ Final Estimate } & 0.53 & 0.045 \\
\hline
\end{tabular}

lung as backscattering from a random collection of small, rigid spheres and results in a technique similar to that used by others, but does not require assumptions of semiregularly spaced scatterers or repeating correlation lengths $[12,13]$. If the broadband backscattered power spectrum were from a single rigid sphere insonified by a broadband pulse of uniform frequency content, its log would have the classical characteristic as shown in figure 4 where the periodicity in $k a$ is approximately 1.3 for $1<k a<5$. Measuring the mode of the power spectrum of figure 4 after the average, or zero frequency component, has been suppressed is one good method of estimating the fundamental periodicity in figure 4. Note that the power spectrum of the log of the power spectrum is identical to the power cepstrum [14]. Generalizing from the model of backscattering from a single rigid sphere to that of backscattering from a distribution of randomly located spheres under the assumption that only first order scattering is significant leads to the estimate of the backscattered power spectrum, i.e.,

where

$$
F|B(f)|=\int_{\infty}^{+\infty} B(k a) p(a) d a,
$$

$$
k=2 \pi / \lambda \text {, }
$$

$a=$ scatterer radius,

$p(a)=$ radii probability density function,

$E[B(f)]=$ estimate of the backscattered power spectrum.

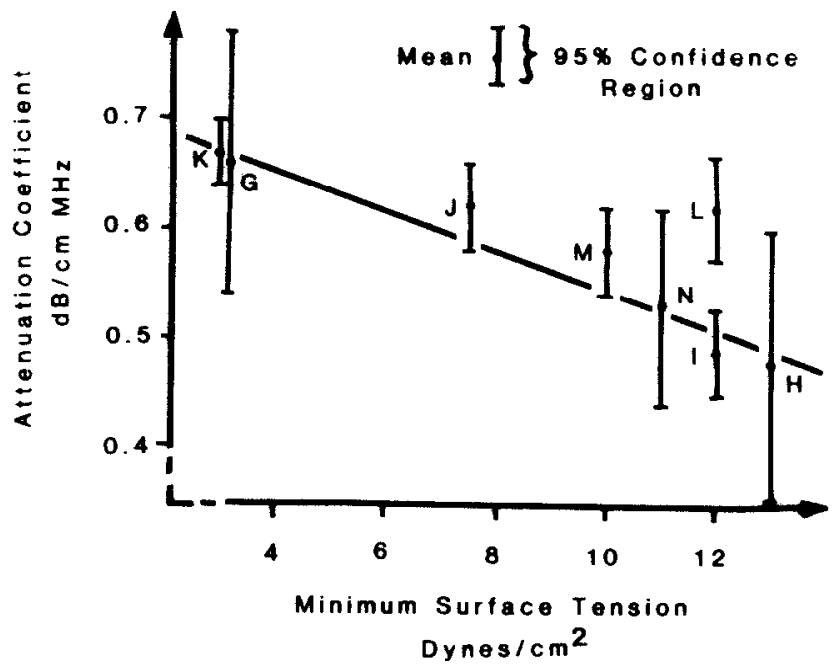

Fig. 3 Linear regression of fetal lung attenuation coefficient estimates with respect to minimum surface tension for eight sheep. 


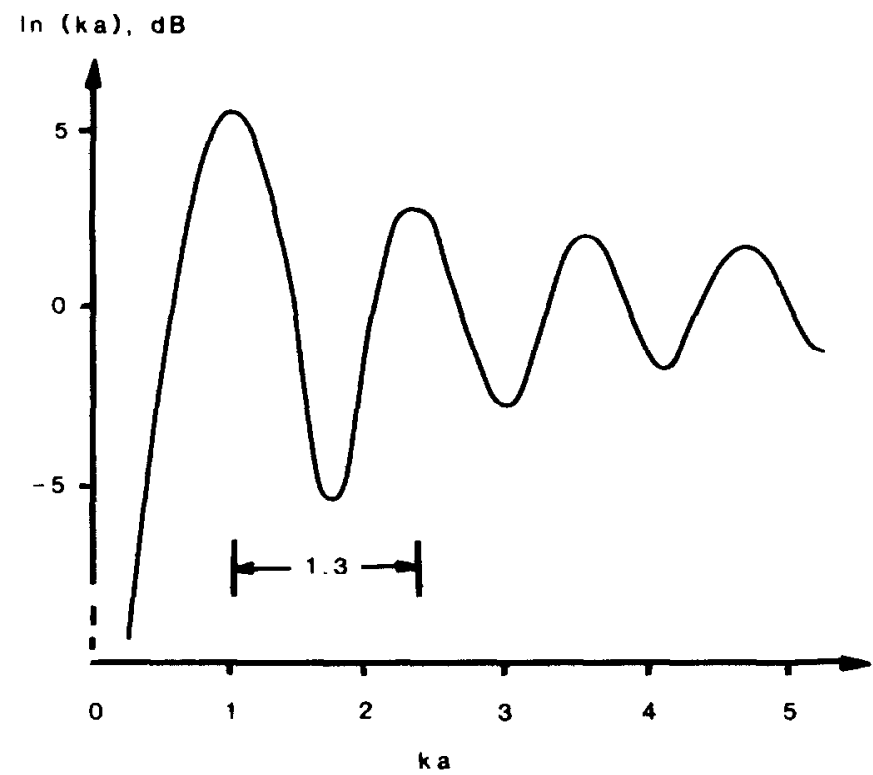

Fig. 4 Logarithm of theoretical backscattered coefficient for a single, rigid sphere of radius a for wavenumber $\mathrm{k}$ where $k=2 \pi / \lambda$.

Note that as $\mathrm{p}(\mathrm{a})$ approaches $\delta\left(\alpha-a_{0}\right), \mathrm{E}[\mathrm{B}(\mathrm{f})]$ approaches $B\left(k a_{0}\right)$ or $B\left(2 \pi f a_{0} / c\right)$ which is the case for the single sphere previously discussed. Figure 5 illustrates the effects of radii variance, $\sigma^{2}$, for 2 cases of $\sigma=0$ and $0.3 a_{0}$ where $a_{0}$ is the mean radius of the distribution. Note that as the variance becomes larger, the amplitude of the fundamental periodicity becomes reduced, but its fundamental period is unchanged.

There are at least two ways of estimating $B(f)$. One involves the use of the previously computed global attenuation coefficient, $\alpha$, to correct for nonstationarity caused by frequency dependent attenuation. In this approach, each segment's power spectrum is multiplied by $e^{4 \alpha n \Delta x f}$ so that the attenuation effects in the last factor of Eq. (1) are nullified. Under the assumption of nearly constant attenuation from overlying tissues between the transducer and the sorted data set, all of the power spectra from each of the segments may be averaged to obtain the estimate

$$
E\left[\ln P_{L a}(f)\right]=\ln g+\ln T^{2}(f)+\ln B(f)
$$

where $P_{L a}$ denotes the received, attenuation-corrected power spectrum for the lung. An alternative approach which involves no assumptions regarding either constant attenua-

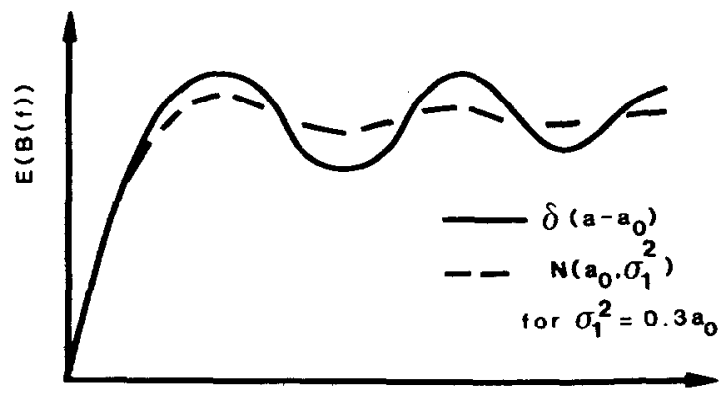

f, trea.
Fig. 5 Effect of population variance in distribution of radii of spherical scatterers on backscattered power spectrum. 
tion of overlying tissues or use of a correct estimate of lung attenuation, averages the logarithms of the power spectra of all segments. In this approach, the nonstationarity due to attenuation is known a priori to result in a term proportional to frequency, i.e., the term $\mathrm{mf}$ in the sum

$$
\ln g+\ln T^{2}(f)+\ln B(f)+m f
$$

which can be estimated by linear regression and then subtracted from the overall estimate again resulting in Eq. (3). While the latter approach is more robust and decouples the cepstral results from the previous global attenuation estimation process, averaging $\log$ spectra yields a slightly larger variance compared to taking the log of averaged power spectra [15]. In comparing the two techniques using the experimental data, we found no significant difference between the two techniques. In order to obtain an estimate of In $\mathrm{B}(f)$ from $E\left[\ln . P_{L a}(f)\right]$, it is necessary to estimate and subtract the effect of the transducer, $\ln T^{2}(f)$. This was accomplished by first acquiring the backscattered signal from a tissue-equivalent phantom made of a material similar to that currently used by RMI using the same transducer and digitizing depth [16]. The attenuation-corrected estimate of backscattering from the RMI phantom was computed by first correcting each segment's spectrum for attenuation using the manufacturer's supplied value and then averaging corrected power spectra to yield

$$
E\left[P_{R M I a}(f)\right]=g_{R M I} T^{2}(f) B_{R M I}(f) e^{-4 b f}
$$

where $b$ is due to material overlying the region of acquired data. The difference between the logs of the power spectral estimates yields

$$
E\left[\ln P_{I \boldsymbol{a}}(f)\right]-E\left[\ln P_{R M / a}(f)\right]=g_{1}+g_{2} f+\ln B_{L}(f)-\ln B_{R M I}(f)
$$

Since the principal scatterers within the RMI material are $6 \mu \mathrm{m}$ graphite particles, the backscattering over the 2 to $6 \mathrm{MHz}$ band where the speed of propagation is $1540 \mathrm{~m} / \mathrm{s}$ is a Rayleigh process and thus can be modeled as $B_{R M I}(f)=g_{\mathrm{S}} f^{4}$. Rewriting and substituting yields

$$
\left(g_{1}-g_{3}\right)+g_{2} f+\ln B_{l}(f)=E\left[\ln P_{7 \boldsymbol{a}}(f)\right\rceil-E\left[\ln P_{R A / \boldsymbol{a}}(f)\right]+4 \ln f .
$$

Since we assume that $\ln B_{L}(f)$ is periodic and has no average slope over a large frequency range, we will estimate $g_{2}$ by linear regression on the sum of terms making up the right side of Eq. (7) and then subtract $g_{2} f$ from the sum to yield

$$
\left(g_{1}-g_{3}\right)+\ln B_{L}(f)=E\left[\ln P_{L a}(f)\right]-E\left[\ln P_{R M I a}(f)\right]+4 \ln f-g_{2} f
$$

To estimate mean backscatterer radius via the relationship demonstrated in figure 4 , i.e. $\Delta k a=1.3$ or

$$
a=\frac{1.3}{2 \pi} c \Delta t
$$

where $\mathrm{a}$ is the radius and $\mathrm{c}$ is the speed of propagation of ultrasound, we need only estimate the periodicity, $\Delta t$, of $\ln B_{L}(f)$. One method of estimating $\Delta t$ is to search for the loci of the global maximum, or mode, of the power spectrum of $\ln B_{L}(f)$, i.e. the power cepstrum, after the average value is removed. The cepstrum is computed by first removing the average value of the sum of terms on the right side of Eq. (B), applying a Hamming window over the region of good signal-to-noise ratio, 2 to $6 \mathrm{MHz}$, computing the fast Fourier transform of the windowed spectrum and multiplying the result by its complex conjugate. Note that when using smoothing windows it is important to remove the average value of the function over the intended length of the window before applying the 
window weighting function. Proceeding in the opposite order guarantees that the value of the resulting power cepstrum is zero for $t=0$, but causes cepstral values for small times near zero to be the difference between the discrete Fourier transform of the rectangular average window (sinc function) and that of the smooth Hamming window. Since the sinc function has significant sidelobes compared to those of the Hamming window transform, large artifactual peaks can be introduced near the ordinate in the cepstrum which may dominate other structures in the true spectrum depending on the magnitude of the original average offset. The recommended approach of subtracting the mean before applying the window introduces no artifical peaks and yields cepstral values of zero for $t=0$ if there are no frequency components in the original log spectrum between zero and the resolution width of the Hamming window transform. Since the resulting power cepstrum is an even periodic function, its value at $t=0$ represents the superposition of the tails of the Hamming window transform from symmetrically located components above and below $t=0$. The superposition of tails for components near $t=0$ introduces a negative bias in the position of the measured mode, which can be corrected if desired. This process of computing a power cepstrum was repeated for each of eight sheep. Figures 6 and 7 demonstrate intermediate rcsults in data processing for sheep N. Figure 8 shows

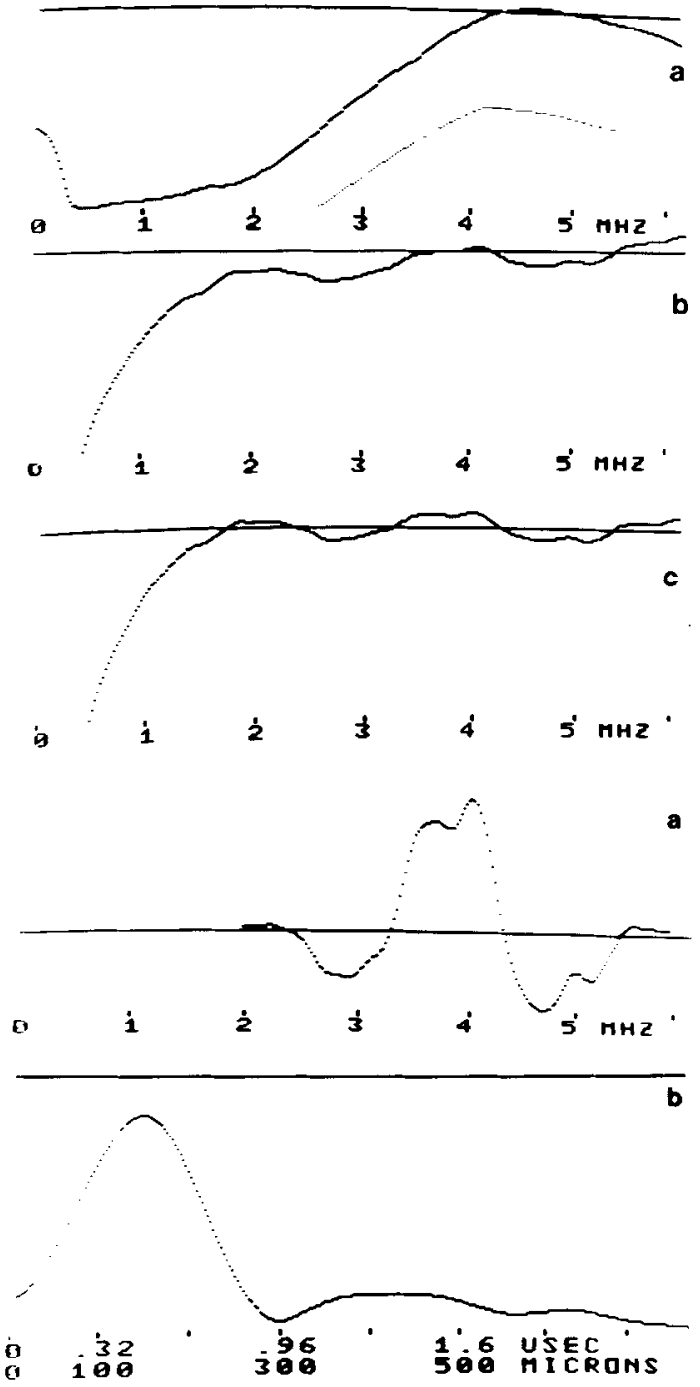

Fig. 6 For fetal sheep N: a) Plots of the logarithms of the attenuationcorrected backscattered power spectra for lung (brighter) and tissue-equivalent phantom (darker). b) Plots of the difference of the two logarithms in $6 a$ plus the term 4 in $f$. c) The result of estimating the straight line slope of $6 b$ via linear regression over the 2 to 6 $\mathrm{MHz}$ range and then subtracting that line. In addition, the average value of the result has been zeroed over the same frequency range. This plot corresponds to the estimate of $\ln B_{L}(f)$ via Eq. 8. (Note correspondence to the theoretical plot in figure 5.)

Fig. 7 For fetal sheep N: a) Hamming window of $6 \mathrm{c}$ over the 2-6 $\mathrm{MHz}$ range. b) Power cepstrum of lung backscattering coefficient obtained by taking the power spectrum of $7 \mathrm{a}$. The mode of the plot corresponds to $455 \mu \mathrm{s}$ or a mean radius of $145 \mu \mathrm{m}$. 


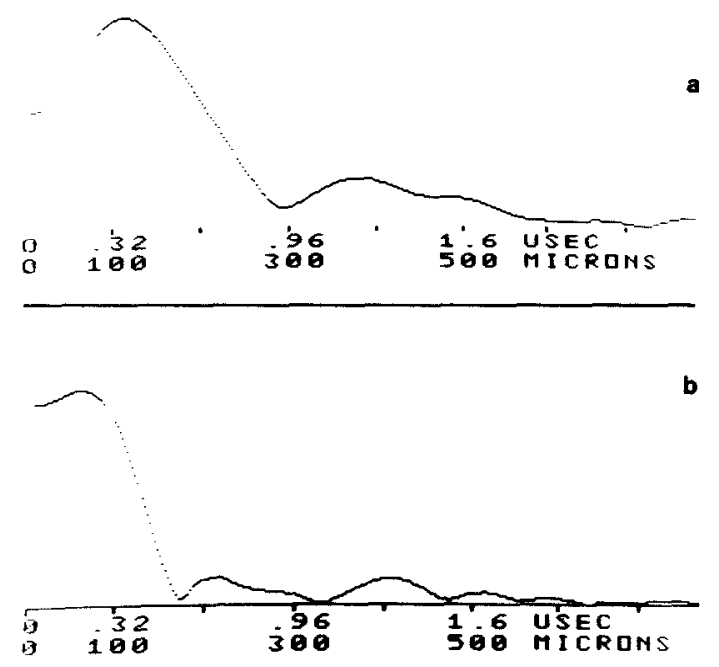

Fig. 8 a) Power cepstrum of lung backscattering coefficient for sheep $G$. b.) Power cepstrum of lung backscattering coefficient for sheep 1.

the cepstral results for sheep $G$ and 1 . Table 2 relates the computed dependent variable of attenuation and the mode of the cepstral peak to the independent parameters of gestational age, mimimum lung surface tension, and visual interpretation by radiologists.

\section{DISCUSSION}

It is not possible to make many significant conclusions from data on only eight fetal sheep, but the trend in the attenuation data certainly points to the possibility of a correlation between functional lung maturity and ultrasonic attenuation. The cepstral results are less reassuring since two outliers must be overlooked to obtain good correlation with functional maturity (under the assumption that larger mian scatterer size is correlated with increased maturation). It is worth noting that cepstral results did follow the visual findings reasonably well. A much larger patient study is required to look into the several possible hypotheses remaining unanswered or generated by this study.

Some preliminaly conclusions can be reached. Due to the stochastic nature of fetal lung backscattering, many independent "views" of the lung must be recorded in order to achieve useful statistical confidence in the attenuation and cepstral results. Nearly 5 MBytes of unsorted $\mathrm{rf}$ data per sheep were required to obtain an attenuation estimate whose 95 percent confidence limits were $\leq 0.2 \mathrm{~dB} / \mathrm{cm} / \mathrm{MHz}$ using a broadband tech-

TABLE 2: COMPARATIVE FINDINGS (Ranking by Attenuation Coefficient)

\begin{tabular}{|c|c|c|c|c|c|c|}
\hline \multirow[b]{3}{*}{$\begin{array}{l}\text { Sheep } \\
\text { Id }\end{array}$} & \multicolumn{3}{|c|}{ Independent Variables } & \multicolumn{3}{|c|}{ Experimental Results } \\
\hline & \multicolumn{2}{|c|}{ Functional Maturity } & \multirow[b]{2}{*}{$\begin{array}{c}\text { Gesta- } \\
\text { tional } \\
\text { Age } \\
\text { (days) }\end{array}$} & \multirow{2}{*}{$\begin{array}{l}\text { Visual } \\
\text { Results } \\
\underset{x \times}{ }\end{array}$} & \multicolumn{2}{|c|}{ Quantitative Results } \\
\hline & $\begin{array}{c}\text { Minimum } \\
\text { Surface } \\
\text { Tension } \\
\left.\text { (dynes } / \mathrm{cm}^{2}\right)\end{array}$ & $\begin{array}{c}\text { Opened at } \\
30 \mathrm{~cm} \text { of } \\
\text { Water } \\
\text { Pressure } \\
\end{array}$ & & & $\begin{array}{l}\text { Atten } \pm 2 \text { SEM } \\
(\mathrm{dB} / \mathrm{cm} \mathrm{MHz})\end{array}$ & $\begin{array}{l}\text { Abcissa of } \\
\text { Cepstral } \\
\text { Peak } \\
\text { (migrons) }\end{array}$ \\
\hline $\mathbf{K}$ & 3.0 & Yes & 145 & $\mathbf{M}$ & $0.67 \pm 0.03$ & 110 \\
\hline $\mathbf{G}$ & 3.0 & Yes & 145 & $\mathbf{M}$ & $0.66 \pm 0.12$ & 110 \\
\hline $\mathfrak{J}$ & 7.6 & Yes & 145 & $\mathbf{M}$ & $0.62 \pm 0.04$ & 115 \\
\hline$L^{\star}$ & 12.0 & No & 145 & $\mathrm{~T}$ & $0.62 \pm 0.05$ & 100 \\
\hline $\mathbf{M}$ & 10.0 & No & 122 & $\mathrm{~T}$ & $0.58 \pm 0.04$ & 160 \\
\hline $\mathbf{N}$ & 11.0 & No & 123 & $\mathbf{M}$ & $0.53+0.09$ & 145 \\
\hline I & 12.0 & No & 125 & I & $0.49 \pm 0.04$ & 55 \\
\hline $\mathrm{H}$ & 13.0 & No & 126 & 1 & $0.48 \pm 0.12$ & 25 \\
\hline
\end{tabular}

* Meconium stained, growth retarded; *x M-Mature, T-Transitional, I-Immature 
nique. Under these conditions arguments of whether attenuation is dependent on $f^{10}$ or $f^{12}$ are moot since the variance in the data swamps effects caused by such small model changes.

\section{ACKNOWLEDGEMENT}

This work was supported by grants from Philips Ultrasound Corp and the National Institute of Human Growth and Development, 1RO1-HD17243-01, National Institutes of Health, DHHS.

\section{REFERENCES}

[1] Gluck, L., Kulovich, M., Fetal lung development: current concepts, Pediatr. Clin. North Am. 20, 367-379 (1973).

[2] Freer, D.E., Statland, B.E., and Sher, G, Quantitation of disaturated phosphatidylcholine and phosphatidylglycerol in amniotic fulid by fluorescence diminuation: methodology and clinical results, Clin. Chem. 25, 960-968 (1979).

[3] Orzalesi, M.M., Motoyama, E.K., and Jacobson, H.N., The development of the lungs of lambs, Pediatrics 35, 373-374 (1965).

[4] Brumley, G.W., Chernick, V., Hodson, W.A., Normand, I.C.S., Fenner, A., and Avery, M.E., Correlations of mechanical stability, morphology, pulmonary surfactant and phospholipid content in the developing lamb lung, $J$. Clin. Invest. 16, 863-873 (1967).

[5] Humphreys, P.W., Normand, I.C.S., Reynolds, E.O.R., and Strang, L.B., Pulmonary lymph flow and teh uptake of liquid from the lungs of the lamb at the start of breathing, $J$. Physiol. (Lond) 193, 1-29 (1967).

[6] Garrett, W.J., Warren, P.S., and Picker, R.H., Maturation of the Fetal Lung, Liver and Bowel, in Proc. Am. Inst. Ultras. in Med. p. 93 (1980) : Abstract only).

[7] Thieme, G.A., Banjavic, R.A., Johnson, M.L., Meyer, C.R., Silvers, G.W., Herron, D.S., and Carson, P.L., Sonographic identification of lung maturation in the fetal lamb, Invest Radiol 18, 18-26 (1983).

[8] Banjavic, R.A., Thieme, G.A., Carson, P.L., Meyer, C.R., and Krasovec, J.J., A 25 MHz Digital Acquisition System and Application to Diagnostic Ultrasound, in 1981 IEEE Ultrasonics Symp. Proc. pp. 597-598 (IEEE Cat. No. 81CH1689-9).

[9] Welch, P.D., The use of fast Fourier transform for the estimation of power specta: a method based on time averaging over short modified periodograms, IEEE Trans Audio Electro Acoustics 15, 70-73 (1967).

[10] Oppenheim, A.V., and Shafer, R.W., Digital Signal Processing (Prentice-Hall, New Jersey, 1975).

[11] Kuc, R., and Schwartz, M., Estimating the acoustic attenuation coefficient slope for liver from reflected ultrasound signals, IEEE Trans. Sonics and Vltrason. 26 , 353-362 (1979).

[12] Rhyne, T.L., and Sagar, K.B., Ultrasonic tissue characterization to diagnose pulmonary embolism, Ultrasonic Imaging 4, 180 (1982) (Abstract only).

[13] Green, S., Joynt, L.F., Fitzgerald, P.J., Rubenson, D.S. and Popp, R.L., In vivo ultrasonic tissue characterization of human intracardiac masses, $\mathrm{Am}$. J. Cardiol. 51 , 231-236 (1983).

[14] Childers, D.G., Skinner, D.P., and Kemerait, R.C., The cepstruin: a guide to processing, Proc. IEEE 65, 1428-1443 (1977).

[15] Stockham, T.G., Cannon, T.M., and Ingebretsen, R.B., Blind deconvolution through digital signal processing, Proc IEEF; 63, 678-692 (1975).

[16] Madsen, E.L., Zagzebski, J.A., Banjavic, R.A., and Jutila, R.E., Tissue mimicking materials for ultrasound phantoms, Medical Physics 5, 391-394 (1978). 